\title{
EULER'S $\phi$-FUNCTION AND SEPARABLE GAUSS SUMS
}

TOM M. APOSTOL

1. Introduction. Let $k$ denote a fixed positive integer. A completely multiplicative arithmetical function $\chi$ is called a character modulo $k$ if $\chi$ is periodic with period $k$ and has the property that $\chi(n)=0$ if and only if $(n, k)>1$. It is well known that there are exactly $\phi(k)$ distinct characters modulo $k$ and that they form a multiplicative group, the identity element being the principal character $\chi_{1}$, where $\chi_{1}(n)=1$ if $(n, k)=1$. Here $\phi(k)$ is Euler's totient.

A positive divisor $d$ of $k$ is called an induced modulus for $\chi$ if we have

$$
\chi(n)=1 \quad \text { whenever }(n, k)=1 \text { and } n \equiv 1(\bmod d) .
$$

This implies that $\chi$ is also a character modulo $d$. In particular, $k$ itself is always an induced modulus for $\chi$. The smallest induced mcdulus is called the conductor of $\chi$. A character $\chi$ modulo $k$ is called primitive if its conductor is $k$, that is, if it has no induced modulus less than $k$.

For any character $\chi$ modulo $k$ and any integer $r$ we consider the Gauss sum $G(r, \chi)$ defined by the equation

$$
G(r, \chi)=\sum_{h \operatorname{lnod} k} \chi(h) e^{2 \pi i r h / k},
$$

where the sum is extended over any complete residue system modulo $k$. We call the Gauss sum separable if we have

$$
G(r, \chi)=\bar{\chi}(r) G(1, \chi) .
$$

It is well known that the Gauss sum $G(r, \chi)$ is separable if $\chi$ is a primitive character (see Lemma 3 below). This paper proves the converse. That is, if $G(r, \chi)$ is separable for every $r$, then $\chi$ is primitive. Therefore, we have the following alternate description of primitive characters.

Theorem 1. A character $\chi$ modulo $k$ is primitive if, and only if, the Gauss sum $G(r, \chi)$ is separable for every $r$.

2. Lemmas. The proof of Theorem 1 is based on seven lemmas. Lemma 6 describes a property of the Euler $\phi$-function which is crucial to the proof of Theorem 1 and also has applications elsewhere [1], $[3$, p. 24], [5, p. 66]. The other lemmas deal with characters and Gauss sums.

Received by the editors June 11, 1969. 
Lemma 1. For any character $\chi$ modulo $k$, the Gauss sum $G(r, \chi)$ is separable whenever $(r, k)=1$.

Proof. Since $(r, k)=1$ the numbers $r h$ run through a complete residue system modulo $k$ with $h$. Also, $|\chi(r)|=1$ so we have $\chi(h)$ $=\bar{\chi}(r) \chi(r) \chi(h)=\bar{\chi}(r) \chi(r h)$. Hence we can write

$$
\begin{aligned}
G(r, \chi) & =\sum_{h \bmod k} \chi(h) e^{2 \pi i r h / k}=\bar{\chi}(r) \sum_{h \bmod k} \chi(r h) e^{2 \pi i r h / k} \\
& =\bar{\chi}(r) \sum_{m \operatorname{macd} k} \chi(m) e^{2 \pi i m / k}=\bar{\chi}(r) G(1, \chi) .
\end{aligned}
$$

This proves that $G(r, \chi)$ is separable.

Lemma 2. Assume $(r, k)>1$. Then $G(r, \chi)$ is separable if and only if $G(r, \chi)=0$.

Proof. If $(r, k)>1$ we have $\bar{\chi}(r)=0$ so equation (3) holds if and only if $G(r, \chi)=0$.

Lemma 3. If $\chi$ is a primitive character modulo $k$, then the Gauss sum $G(r, \chi)$ is separable for every $r$.

Proof. A proof of Lemma 3 is given in [2, Theorem 4.12, p. 312] and in $[4$, Lemma 1.1, p. 212].

Lemma 3, together with its converse (Lemma 7 below) give us Theorem 1. The next three lemmas are used to prove Lemma 7.

Lemma 4. If $\chi$ is a primitive character $\bmod k$, then $|G(1, \chi)|^{2}=k$.

Proof. A proof of Lemma 4 is given in [2, Theorem 4.13, p. 313] and in [4, Lemma 1.1, p. 212].

LEMMA 5. Let $\chi$ be any character modulo $k$ and let $d$ be the conductor of $\chi$. Then there exists a primitive character $\psi$ modulo $d$ such that

$$
\chi(n)=\psi(n) \chi_{1}(n),
$$

where $\chi_{1}$ is the principal character modulo $k$.

Proof. We define $\psi(n)$ by the equation $\psi(n)=\chi(n) / \chi_{1}(n)$ if $(n, d)=1$ and we let $\psi(n)=0$ if $(n, d)>1$. Then equation (4) holds for all $n$. It is easy to verify that $\psi$ is a character modulo $d$. To prove that $\psi$ is a primitive character modulo $d$, let $q$ be any induced modulus for $\psi$. Then we have

$$
\psi(n)=1 \quad \text { if }(n, d)=1 \text { and } n \equiv 1(\bmod q) .
$$

Equation (4) implies that $\chi(n)=1$ if $(n, k)=1$ and $n \equiv 1(\bmod q)$, so $q$ is also an induced modulus for $\chi$. Hence $q \geqq d$ since $d$ is the conductor 
of $\chi$. Therefore the conductor of $\psi$ is equal to $d$ so $\psi$ is primitive modulo $d$. This proves Lemma 5 .

The next lemma concerns decomposition of reduced residue systems.

Lemma 6. Let $S_{k}$ denote a reduced residue system modulo $k$, and let $d$ be a divisor of $k$. Then $S_{k}$ is the union of $\phi(k) / \phi(d)$ disjoint sets, each of which is a reduced residue system modulo $d$.

Proof. Consider $S_{k}$ as a multiplicative group of reduced residue classes modulo $k$, and let $S_{d}$ be the group of reduced residue classes modulo $d$. Let the classes of $S_{k}$ be represented by integers $n$ and those of $S_{d}$ by integers $r$, and note that each $n$ is congruent $(\bmod d)$ to a number $r$ since $d \mid k$. Define a mapping $f: S_{k} \rightarrow S_{d}$ as follows:

$$
\text { If } n \in S_{k}, \quad \text { then } f(n)=r \text {, where } n \equiv r(\bmod d) \text {. }
$$

This mapping is a homomorphism of $S_{k}$ into $S_{d}$. The homomorphism is onto because if $(r, d)=1$ there always exists an integer $n$ such that

$$
n \equiv r(\bmod d) \quad \text { and }(n, k)=1 \text {. }
$$

In fact, we can take for $n$ the solution to the system of congruences

$$
x \equiv r(\bmod d), \quad x \equiv 1\left(\bmod k^{\prime}\right),
$$

where $k^{\prime}$ is the product of those prime factors of $k$ which do not divide $d$. Since $\left(k^{\prime}, d\right)=1$ this system has a solution (by the Chinese remainder theorem). To prove that $(n, k)=1$ we note that $\left(n, k^{\prime}\right)=1$ because $n \equiv 1\left(\bmod k^{\prime}\right)$ and that $(n, d)=1$ because $n \equiv r(\bmod d)$. Hence $\left(n, k^{\prime} d\right)=1$. But $k$ and $k^{\prime} d$ have the same set of prime factors, so $(n, k)=1$.

Now let $K$ be the kernel of $f$, that is, $K=\left\{x \in S_{k} \mid x \equiv 1(\bmod d)\right\}$. Then the factor group $S_{k} / K$ is isomorphic to the group $S_{d}$, so we have a corresponding coset decomposition

$$
S_{k}=\bigcup_{x \in T} x K,
$$

where $T$ is a set of coset representatives. If we take one representative from each coset we get a reduced residue system modulo $d$. There are $\phi(k)$ elements in $S_{k}$ and $\phi(d)$ elements in each reduced residue system modulo $d$, so there are $\phi(k) / \phi(d)$ such residue systems altogether. This completes the proof of Lemma 6 .

Note. The referee has pointed out that Lemma 6 was proved in 1923 by T. Nagell [3], and that a different proof was later given by R. Vaidyanathaswamy [5]. Our group-theoretic proof is different from each of these. 
Now we use Lemmas 4, 5, and 6 to prove the converse of Lemma 3.

LEMMA 7. If a character $\chi$ modulo $k$ has separable Gauss sums $G(r, \chi)$ for every $r$, then $\chi$ is primitive modulo $k$.

Proof. Because of Lemmas 1 and 2, it suffices to prove that if $\chi$ is not primitive then for some $r$ satisfying $(r, k)>1$ we have $G(r, \chi) \neq 0$. Suppose, then, that $\chi$ is not primitive modulo $k$. This implies $k>1$. Then $\chi$ has a conductor $d<k$. If $d=1$ then $\chi=\chi_{1}$ and we have

$$
G\left(r, \chi_{1}\right)=\sum_{\Lambda \bmod k} \chi_{1}(h) e^{2 \pi i r h / k}=\sum_{h \bmod k ;(h, k)=1} e^{2 \pi i r h / h} .
$$

When $r=k$ we have $G\left(k, \chi_{1}\right)=\phi(k) \neq 0$. This proves the lemma for the case in which the conductor $d=1$.

Now suppose $d>1$ and let $r=k / d$. We have $(r, k)>1$ and we shall prove that $G(r, \chi) \neq 0$ for this $r$. By Lemma 5 there exists a character $\psi$ modulo $d$ such that $\chi(n)=\psi(n) \chi_{1}(n)$ for all $n$. Hence we can write

$$
\begin{aligned}
G(r, \chi) & =\sum_{h \bmod k} \psi(h) \chi_{1}(h) e^{2 \pi i r h / k}=\sum_{h \bmod k ;(h, k)=1} \psi(h) e^{2 \pi i r h / h} \\
& =\sum_{h \bmod k ;(h, k)=1} \psi(h) e^{2 \pi i h / d}=\frac{\phi(k)}{\phi(d)} \sum_{h \bmod d_{i}(h, d)=1} \psi(h) e^{2 \pi i h / d},
\end{aligned}
$$

where in the last step we used Lemma 6 . Therefore we have

$$
G(r, \chi)=\frac{\phi(k)}{\phi(d)} G(1, \psi) .
$$

But $|G(1, \psi)|^{2}=d$ by Lemma 4 (since $\psi$ is primitive modulo $d$ ) and hence $G(r, \chi) \neq 0$. This completes the proof of Lemma 7. As already mentioned, Lemmas 3 and 7 together prove Theorem 1.

\section{REFERENCES}

1. Tom M. Apostol, Resultants of cyclotomic polynomials, Proc. Amer. Math. Soc. 24 (1970), 457-462.

2. Raymond Ayoub, An introduction to the analytic theory of numbers, Mathematical Surveys, no. 10, Amer. Math. Soc., Providence, R. I., 1963. MR 28 \#3954.

3. Trygve Nagell, Zahlentheoretische Notizen, Skr. Norske Vid. Akad. Oslo I 13 (1923), 23-25.

4. Karl Prachar, Primzahlverteilung, Springer-Verlag, Berlin, 1957. MR 19, 393.

5. R. Vaidyanathaswamy, $A$ remarkable property of the integers $\bmod n$ and its bearing on group theory, Proc. Indian Acad. Sci. Sec. A 5 (1937), 63-75.

California Institute of Technology 